\title{
LÁSER Y TUMORES VESICALES
}

\author{
J. Rioja Zuazu, V. Toutziaris, M. P. Laguna Pes, T. M. de Reijke y J. J. de la Rosette.
}

Academic Medical Center. University of Amsterdam. Amsterdam. TheNetherlands.

Resumen.- Los tumores vesicales suponen una de las enfermedades más frecuentes de la urología. La introducción de los láseres en el armamentario terapéutico urológico supuso un gran avance, pero los resultados iniciales con los primeros modelos, no alcanzaron las expectativas. Sin embargo, en la actualidad disponemos de una gran variedad de láseres para uso urológico, con distintas funciones y aplicaciones. Realizamos una revisión e intentamos asentar las indicaciones actuales de su utilización en el tratamiento de los tumores vesicales, así como una visión de futuro de sus posibles aplicaciones.

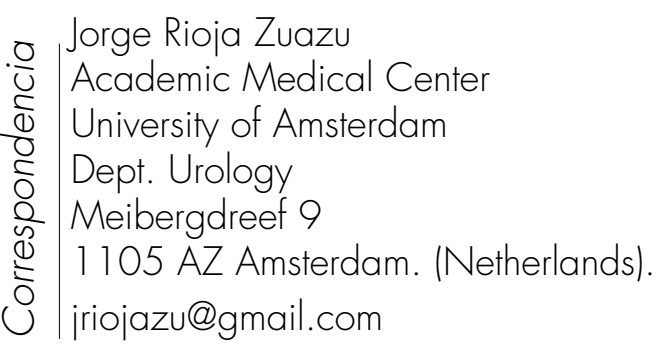

Palabras clave: Cáncer de vejiga. Fulguración láser. Láser.

Summary.- Bladder cancer is one of the most frequents pathologies in urology. The introduction of the laser among the technical tools supposed a great advance, but the initial results with the first model failed with the expectations. Nevertheless, nowadays we have at our disposal a great variety of laser for urological use, with different functions and applications. We perform a review and try to assess the actual indications for its employment in the treatment of bladder cancer, as well as future applications.

Keywords: Bladder cancer. Laser fulguration. Laser.

\section{INTRODUCCIÓN}

El cáncer de vejiga supone el cuarto tumor más diagnosticado en el hombre y el octavo más común en la mujer en USA (1), y aproximadamente un $75-85 \%$ de los casos se presenta como enfermedad confinada a la mucosa (Ta-CIS) o a la submucosa (T1) (2).

En los caso de tumores múltiples, grandes, y de alto riesgo (TaG3, TIG3, CIS) es preciso, en ocasiones, realizar una re-resección para asegurar la correcta estadificación del mismo. Ocasionalmente se realizan re-resecciones por una primera resección incompleta o insuficiente (3). La necesidad de disminuir la morbilidad de estos procedimientos, así como de su coste, nos ha llevado a invertir en tratamientos mínimamente invasivos.

La energía láser, resulta fácil de aplicar y de una manera efectiva, con una probable disminución de la morbilidad. En el campo de la cirugía urológica, los láseres se están convirtiendo en una herramienta cada vez más importante. La tecnología láser se ha aplicado con éxito en 
una gran variedad de condiciones benignas que incluyen patología litiásica, hiperplasia benigna de próstata, verrugas genitales y en lesiones angiomatosas

De manera adicional, se ha utilizado a su vez para el tratamiento de determinadas enfermedades malignas como los tumores de urotelio o los carcinomas de células escamosas (4).

El objetivo de este artículo es revisar los principios básicos de la terapia con láser, establecer su papel en el tratamiento del carcinoma de células transicionales de vejiga y revisar las últimas novedades de esta tecnología en este campo.

\section{Principios de la energía láser y sus efectos tisu- lares}

Los láseres quirúrgicos tienen por objetivo excitar átomos en un medio con el resonador del láser (5), generando un flujo de energía. Este flujo de energía permite a los átomos alcanzar niveles de energía más altos y liberar fotones de luz conforme vuelven a su estado de reposo. Estos fotones son recogidos, amplificados, focalizados y canalizados a lo largo de una serie de espejos o de una fibra flexible. La longitud de onda de un determinado láser está predeterminada por el medio activo que se utiliza para generar los fotones.

La respuesta tisular, por contra esta determinada por las características crómicas de las células que absorben selectivamente distintas longitudes de onda de la luz. Además, la cantidad de energía liberada en un sitio específico (densidad de energía) depende, de la duración de la exposición a la energía, así como la capacidad de la máquina de entregar la energía en un campo reducido (ángulo de divergencia).

La mayoría de los láseres utilizados hoy en día son capaces de focalizar la energía con unos ángulos de divergencia entre $5^{\circ}$ y $15^{\circ}$. Aunque este foco permite una liberación más eficiente de la energía, también incrementa el riesgo de una lesión térmica en las estructuras adyacentes, debido a la exposición a alta densidad de energía que se puede extender a lo largo de varios centímetros. El daño tisular es consecuencia de la liberación de la energía térmica a las células expuestas (6). La temperatura necesaria para producir los cambios irreversibles consecuentes a la desnaturalización proteica y a la coagulación tissular en los tejidos, oscilan entre $60^{\circ} \mathrm{C}$ y $100^{\circ} \mathrm{C}$. La vaporización tisular, se obtiene cuando las temperaturas exceden los $100^{\circ} \mathrm{C}$. Mientras esta temperatura es fácil de alcanzar en la superficie corporal, a nivel vesical y por los efectos de una irrigación de fluido circulando alrededor este objetivo resulta mucho más complejo.

\section{Elección del Láser}

La longitud de onda de la luz emitida por el láser difiere en función del medio empleado para generar la energía fotónica. Múltiples láseres se han desarrollado y utilizado para diversas aplicaciones en urología (Tabla I) (5).

\section{- El Láser Nd-YAG}

Ha sido tradicionalmente el láser más empleado en el campo de la urología. El medio activo es Neodymium que esta contenido en un entramado de Ytrio, Aluminio y Garnet. La luz emitida por este instrumento, tiene una longitud de onda de $1064 \mathrm{~nm}$ y es pobremente absorbida por el agua y los pigmentos tisulares, es por ello que su energía es capaz de alcanzar una profunda penetración tisular. Como resultado, el láser tiende a producir una excelente coagulación termal de la lesión a tratar, tanto en la superficie como por debajo de ella. El tejido tratado, adopta una coloración blanquecina, aterciopelada, que desprende unos días después. La hemostasia obtenida es completa.

Existe un riesgo de daño térmico en los tejidos adyacentes, sobre todo en la cúpula de la vejiga y en vejigas con pared adelgazada, debido a tratamientos previos mediante resecciones trans-uretrales.

TABLA I. LÁSERES UTILIZADOS EN UROLOGÍA.

\begin{tabular}{|c|c|c|c|}
\hline Tipo & Longitud de Onda $(\mathbf{n m})$ & Disponibilidad Endoscópica & Profundidad de penetración (mm) \\
\hline Nd:YAG & 1064 & $\mathrm{Si}$ & $3-5$ \\
\hline KTP:YAG & 532 & $\mathrm{Si}$ & $1-2$ \\
\hline $\mathrm{CO}_{2}$ & 10600 & $\mathrm{No}$ & 0,1 \\
\hline Argón & $488-514$ & $\mathrm{Si}$ & $0,5-1,5$ \\
\hline Ho:YAG & 2100 & $\mathrm{Si}$ & $0,5-1,0$ \\
\hline
\end{tabular}




\section{- El láser KTP Nd-YAG}

Produce energía a través de un cristal de potasio, titanyl y fosfato, produciendo una luz verde, visible al ojo humano, y con una longitud de onda de $532 \mathrm{~nm}$. Sin embargo esta interacción disminuye la potencia efectiva de la energía que puede ser liberada en con un simple disparo. Los $532 \mathrm{~nm}$ de longitud de onda tienen una menor penetración tisular que el láser de $\mathrm{Nd}-\mathrm{YAG}$, aunque este hecho hace que el tratamiento de las lesiones grandes se más tedioso y difícil, presenta una gran seguridad para el tratamiento de las lesiones pequeñas y superficiales (4).

\section{- Los láseres de Argón}

Producen una energía con una longitud de onda entre 488 y $514 \mathrm{~nm}$. La energía que se absorbe de manera muy ávida por los pigmentos como la hemoglobina o la melanina. Este hecho lo convierte en una herramienta muy útil para el tratamiento de hemangiomas cutáneos o en las nefrectomías parciales. Su aplicación en el tratamiento de tumores vesicales ha sido muy limitada, por su bajo rendimiento y no ha encontrado una aceptación adecuada. A su vez este tipo de láser pueden ser utilizados con otros tipos de tinciones para producir energía fotónica con distintas longitudes de onda. Esta especificidad permite al cirujano seleccionar distintos cromoforos tisulares, fundamento de la terapia con hematoporfinas en tumores superficiales y carcinoma in situ de vejiga (7).

\section{- Los láseres de $\mathrm{CO}_{2}$}

Pueden entregar una potente cantidad de energía a determinadas lesiones, causando una vaporización del mismo. Su longitud de onda de $10600 \mathrm{~nm}$ es absorbida por el cristal y el agua. Efectos prácticos han limitado el uso endoscópico del laser de $\mathrm{CO}_{2}$ en la vejiga.

\section{- Holmiun Láser (HoYAG)}

La longitud de onda láser de que disponemos más recientemente con fines quirúrgicos es el Holmiun láser (Ho: YAG). Este láser utiliza un extraño componente de la tierra, el Holmio, en conjunción con un cristal de YAG. Como resultado tenemos una longitud de onda de $2100 \mathrm{~nm}$ invisible al ojo humano y que requiere la adición de una luz a modo de puntero que se emplea para dirigir el haz de láser. Mediante la manipulación de los ajustes de potencia y pulso, este láser es capaz de coagular y vaporizar tejido. Su efecto hemostático resulta excelente, permitiendo al cirujano trabajar en un campo exangüe.

Finalmente, la alta absorción del láser holmiun por el agua, (como con el láser de $\mathrm{CO}_{2}$ ) significa que el tejido superficial sufre la mayoría del efecto térmico, limita la profundidad de la penetración tisular en aproximadamente $0,5 \mathrm{~mm}$ y $1 \mathrm{~mm}$. Su mayor aceptación, ha sido en el campo de la litotricia intracorpórea desde los primeros informes en 1995 (8). Sin embargo su aplicación en el manejo del cáncer de urotelio no ha tenido mucha difusión, aunque algunos autores han presentado su eficacia tanto en el tratamiento de esta patología a nivel vesical y en el tracto urinario superior (9-11).

\section{Láser y tratamiento del cáncer vesical}

\section{Selección de pacientes}

\section{Características}

Las lesiones pequeñas y papilares son las susceptibles de manejar mediante láser. Muchos autores tienen el sentimiento que esta técnica está reservada para aquellos pacientes con una historia previa, con confirmación anatomo-patológica, de tumor de células transicionales de bajo grado no invasivo ( $\mathrm{Ta} / \mathrm{T} 1)$. También se puede realizar una biopsia fría de una lesión sospechosa y emplear el láser como herramienta de hemostasia y erradicar el resto de lesiones.

El empleo del láser en el tratamiento de los tumores superficiales de vejiga, fue realizado por primera vez en Alemania a mediados de los 70's y fue aprobado para su utilización en USA en 1984 (7).

El tratamiento de los tumores vesicales no invasivos mediante láser se realiza normalmente de manera ambulatoria y requiere una anestesia menos profunda que la electrocoagulación convencional. Esto es debido al hecho que la energía láser resulta en un calentamiento más rápido y en una destrucción del tejido y de las fibras nerviosas, mientras que la corriente eléctrica convencional, se retropropaga a lo largo de la fibra nerviosa.

Además, la capacidad de aplicar este tratamiento por medio de una fibra flexible, permite su aplicación a través de la utilización de un cistoscopio flexible, evitando las molestias propias de instrumento rígido. Para aquellos pacientes sometidos a anestesia raquídea o general, un cistoscopio rígido con un puente para la fibra láser permite un excelente control y precisión en el manejo de la fibra.

Otra de las ventajas de emplear este tipo de energía es que se evita el empleo de una sonda vesical. Muchos pacientes no sufren ningún cambio en su orina, mientras que otros refieren un aumento de los sedimentos conforme el tejido coagulado muda. Episodios de sangrados tardíos son excepcionales (7).

Algunos autores han publicado que el empleo de la terapia láser en lesiones grandes, superficiales puede condicionar una menor tasa de recurrencia que con la electrocoagulación convencional (12-14). Aunque este tratamiento probablemente no afecta a la incidencia de recurrencia en porciones no tratadas del urotelio, la transmisión trans-mural de la energía térmica del láser, puede determinar una dosis de radiación más efectiva que la que se puede conseguir con la electrocoagulación.

El tratamiento con láser de los tumores infiltrantes de vejiga se realiza, normalmente, después de una resección transuretral agresiva, con un instrumento rígido, aunque se puede utilizar uno flexible que permite llegar mejor a determinadas áreas de la vejiga. Se reserva únicamente para tratamientos paliativos, en pacientes que por su elevada morbilidad no pueden ser sometidos a una cirugía radical (15) (Tabla II). 


\section{TABLA II. PRINCIPALES INDICACIONES DE LA TERA- PIA LÁSER EN TUMORES DE VEJIGA.}

\section{Principales indicaciones}

- Tumores superficiales con múltiple recidivas

- Tumores superficiales múltiples y pequeños $(<1 \mathrm{~cm}$.)

- Pacientes con riesgo quirúrgico alto

- Tratamiento paliativo en tumores infiltrantes

\section{Ventajas e inconvenientes}

- Las ventajas principales

- Realización ambulatoria

- No necesidad de anestesia

- Menor riesgo de sangrado y mejor coagulación del lecho.

- Posibilidad de realización con instrumento flexible bajo anestesia local.

- No necesidad de sondaje vesical.

- Tratamiento de tumores en el interior de divertículos vesicales con menor riesgo.

- Realización con cualquier tipo de solución para irrigación.

- No estimulación del nervio obturador.

- Posibilidad de fotocoagulación alrededor del meato ureteral.

- Los principales inconvenientes son:

- No biopsia (relativo).

- Dificultad de visualizar la erradicación completa.

- Inversión en aparataje (equipo láser).

\section{Técnica de fotocoagulación}

La realización de una fotocoagulación con láser de un tumor vesical tiene una técnica, así como unos trucos determinados y difiere entre tumores superficiales y profundos.

En tumores superficiales, lo primero que hay que configurar es el ajuste de la energía que va a entregar el equipo

El láser de $\mathrm{Nd}: Y A G$, requiere generalmente una energía para coagular las lesiones en vejiga entre 30 y $40 \mathrm{~W}$. Utilizando una fibra estándar de entre $400-600 \mu \mathrm{m}$, la punta de la misma debe colocarse a unos 3 y $4 \mathrm{~mm}$ de la lesión. La energía se debe aplicar de manera continua como si se estuviese "pintando" la vejiga y el tumor, hasta que el tumor va adquiriendo una coloración blanquecina. En ocasiones, la superficie del tumor parece que está hirviendo, conforme el tejido se coagula por completo. El área coagulada puede ser desprendida con la punta de la fibra para exponer la base del tumor para un tratamiento posterior. Normalmente se necesitan entre 2 y 3 segundos para obtener una coagulación completa del punto tratado. Otra ventaja, es que se puede utilizar cualquier tipo de solución de irrigación para la transmisión de energía. Sistemas de irrigación continua, no son normalmente necesarios porque se consigue una hemostasia completa.

El láser de Holmiun, se aplica de una manera similar que el $\mathrm{Nd}$ :YAG con la ventaja de una penetración más superficial. Por esta razón, puede resultar más seguro para su empleo en los tumores localizados en la cúpula de la vejiga, pero por el contrario, el tratamiento de tumores más grandes resulta tedioso y largo. Requiere un menor voltaje, entre 5 y $15 \mathrm{~W}$, y normalmente se emplea con los siguiente ajustes de energía: 0,5-1,5 Julios/pulso y una frecuencia de pulso de $15 \mathrm{~Hz}$.

Una de las posibilidades que ofrece el láser Ho: YAG es la de realizar una resección vesical (HoLRBT) no existiendo diferencias histopatológicas entre el espécimen obtenido mediante biopsia fría y el obtenido mediante esta técnica (16).

Se debe tratar cualquier parte del tumor o del área de alrededor hasta que torne la típica coloración blanquecina propia de la coagulación, normalmente después de 3, 4 segundos. Resulta de suma importancia tratar toda la base tumoral así como un margen de seguridad de $1 \mathrm{~cm}$, más o menos. Hay que tener mucho cuidado al tratar determinadas áreas vesicales, más delgadas, porque este tejido es más susceptible de transmitir la energía a órganos vecinos dañando dichas estructuras. Esto hecho ocurre sobretodo en la cúpula y en la pared posterior de la vejiga (Figura 1).

Debido a la transmisión de la energía de manera transmural, es importante mantener la vejiga no completamente distendida para evitar el adelgazamiento de la pared evitando la transmisión a estructuras vecinas.

Para el tratamiento de tumores infiltrantes, se emplean unos ajustes distintos que en los tumores superficiales, mayor potencia (entre 40 y $50 \mathrm{~W}$ ) y la energía se entrega de una manera continua. Los principios básicos de esta configuración es que la aplicación de la energía láser en el cráter tumoral puede inducir de manera completa la coagulación del tejido y por ello eliminar cualquier residuo de tumor que pueda quedar en el área tratada.

Además, varias investigaciones llevadas a cabo por Hofstetter y cols. (15) sugerían que la aplicación circunferencial del láser en la base del tumor podría inducir un sellado de los vasos linfáticos peritumorales. El significado clínico de esta teoría sigue siendo desconocido. Se debe tratar cualquier parte del tumor o del área de alrededor, de la misma manera que se hace con los tumores no invasivos, ampliando el margen de seguridad, observando la misma precaución con determinadas áreas de la vejiga. 
Se ha descrito una técnica combinando cistoscopia y laparoscopia, en la que una laparoscopia intraperitoneal se utiliza para ayudar a retraer los intestinos de la pared vesical así como para observar los efectos de la coagulación con láser sobre la pared vesical (17). A su vez la fibra se puede emplear por el lado intraperitoneal para tratar la pared externa de la vejiga y asegurarse de un efecto global en la pared de la vejiga, sin incrementar el riesgo de perforación vesical ya que permanece el cito-esqueleto de la vejiga. Esta técnica se limita en las lesiones localizadas en el trígono o pared posterior-suelo vesical.

Se puede realizar con instrumento flexible o rígido. Con ambos instrumentos hay que mantener la punta fuera del cistoscopio cuando se transmite la energía, debido al riesgo de rotura de la óptica.

Con cistoscopio rígido se debe utilizar una vaina especial para fibra láser o en su defecto pasarlo a través de un catéter ureteral.

Con el cistoscopio flexible se debe tener la misma distancia de seguridad, y además antes de flexionar el cistoscopio la punta de la fibra debe asomar por la punta del cistoscopio para evitar roturas de la fibra dentro del cistoscopio (Figura 2).

\section{Control de calidad}

\section{Resultados}

Analizando los resultados publicados en la literatura encontramos un estudio aleatorizado, Beisland y Seland (13) presentaron una recurrencia del $7 \%$ en tumores $\mathrm{T} 1$ tratados con láser $\mathrm{Nd}$ :YAG frente a $43 \%$ en tumores similares tratados con electrocoagulación estándar. A su vez Beer y cols. (12) presentaron una tasa similar de recurrencia local en una serie de 252 pacientes con fumor superficial tratados con láser de manera consecutiva.

Vicente y cols. (18) comparando 109 pacientes tratados con láser $\mathrm{Nd}$ :YAG con otros sometidos a resec-

\section{TABLA III. PORCENTAJE DE RECURRENCIA TRAS TRA- TAMIENTO CON LÁSER ND:YAG DE LOS TUMORES INFILTRANTES DE VEJIGA (PORCENTAJE).}

\begin{tabular}{|c|c|c|c|}
\hline Series & T2 \% & T3 \% & T4 \% \\
\hline McPhee (23) & 0 & 43 & 100 \\
\hline Smith (24) & 18 & 50 & 100 \\
\hline Beisland (25) & 27 & - & - \\
\hline Media & 16 & 47 & 100 \\
\hline
\end{tabular}

ción convencional, no encontraron diferencias en la tasa de recurrencia o progresión, no hubo cambios en la capacidad vesical, presentando una menor tasa de incidencia de estenosis uretral los pacientes sometidos a tratamiento mediante láser $(4,5 \%)$ frente a los sometidos a resección convencional $(16,3 \%)$, sugiriendo como indicaciones de tratamiento láser la presencia de un fumor único, menor de 2-3 cm. múltiple inicialmente o recurrente.

Estos resultados se repiten con el empleo del láser de Ho:YAG, Syed y cols. (19) presentaron unas tasas de recidiva sensiblemente inferiores con el empleo del láser de Ho:YAG (10\%) frente a la electrocoagulación convencional $(36 \%)$ existiendo diferencias estadísticamente significativas entre ambas $(p=0,39)$, así como una mayor satisfacción del paciente por el tratamiento recibido (menos doloroso) y un menor coste, debido a su realización ambulatoria. Por su parte Soler-Martínez y cols. (20) presentan unos resultados de recurrencia similares a los obtenidos utilizando la resección convencional, estimada mediante la calculadora del riesgo del de recurrencia de la European Association of Urology (EAU). Por su parte Jønler y cols. (21), en un estudio sobre 52 pacientes con tumor vesical superficial, concluye que la vaporización con láser Ho:YAG es factible, fácil y rápida, con un alto porcentaje de satisfacción por parte del paciente, así como una versión positiva desde el punto de vista económico.

En la última puesta al día de la Asociación Americana de Urología (AUA) en el tratamiento de tumores superficiales de vejiga, se acepta la utilización de terapias con energía láser para el tratamiento de determinados tumores del tracto urinario superior e inferior, sobre todo aquellos de bajo grado y papilares, excluyendo los nuevas lesiones sin estadiar (22).

El láser $\mathrm{Nd}$ :YAG ha sido utilizado de manera conjunta con la electrocoagulación para el tratamiento de tumores vesicales infiltrantes $\left(T_{2}, T_{3 a b b}\right.$ y $\left.T_{4}\right)$.

Los resultados de está técnica no son sorprendentes, y ponen de manifiesto las limitaciones de la técnica (23-25). La tasa de recurrencia media, en tumores infiltrantes después de tratamiento con láser $\mathrm{Nd}$ :YAG es, para $\mathrm{T}_{2}$ entorno al $16 \%$, todos los $T_{4}$ presentan recurrencia y para los $\mathrm{T}_{3}$ oscila entre el $25 \%$ y el $100 \%$ (Tabla II). Tarantino y cols. estudiaron el efecto de la terapia con láser sobre el estadio tumoral en pacientes sometidos posteriormente a cistectomía radical, siendo capaces de obtener un estadio patológico correcto en todas las lesiones. Los resultados fueron muy pobres para todos los estadios, incluidos los estadios T2 (26) (Tabla III).

\section{Complicaciones}

Las complicaciones de la foto-coagulación con láser de tumores en la vejiga son muy bajas. La mayoría de los pacientes presentan un cierto grado de disuria inicial que desaparece sin problemas.

La principal complicación es la lesión de órganos adyacentes por lesión térmica, fundamentalmente intestino delgado o colon. Varias series extensas, con más de 2000 
pacientes tratados lo estiman entre un $0,15 \%$ y $0,30 \%(12$, 27). Es importante destacar que esta complicación puede ocurrir en ausencia de perforación vesical. Ante la duda de perforación intestinal es obligatoria la realización de una laparotomía de urgencia.

Otra de las complicaciones más frecuentes es la insuficiente coagulación de la lesión en cuestión por una mala elección de la técnica, es decir, aquellos pacientes con tumores demasiado grandes para ello.

\section{Coste}

Respecto al coste de este tratamiento, existen varios estudios en los que se confirma que es un tratamiento más barato que la RTU convencional, puesto que se realiza de manera ambulatoria. Llegando a ahorrar unos $1350 €$ al hospital para un caso ambulatorio y $350 €$ para casos con un día de estancia (21). Dichos estudios no incluyen el coste del aparato láser.

\section{TERAPIA FOTODINÁMICA (PDF)}

A pesar de los múltiples avances en urología, el tratamiento más efectivo para el tumor vesical superficial sigue siendo la resección transuretral. Sin embargo las múltiples, difusas y frecuentes recurrencias, son más difíciles de controlar por medio de la resección transuretral sola o en combinación con otros tratamientos quimioterápicos o de inmunoterapia intravesical (28). Una técnica que ha sido evaluada por su efecto en los tumores de alto grado así como en los tumores recurrentes, en un intento de prevenir
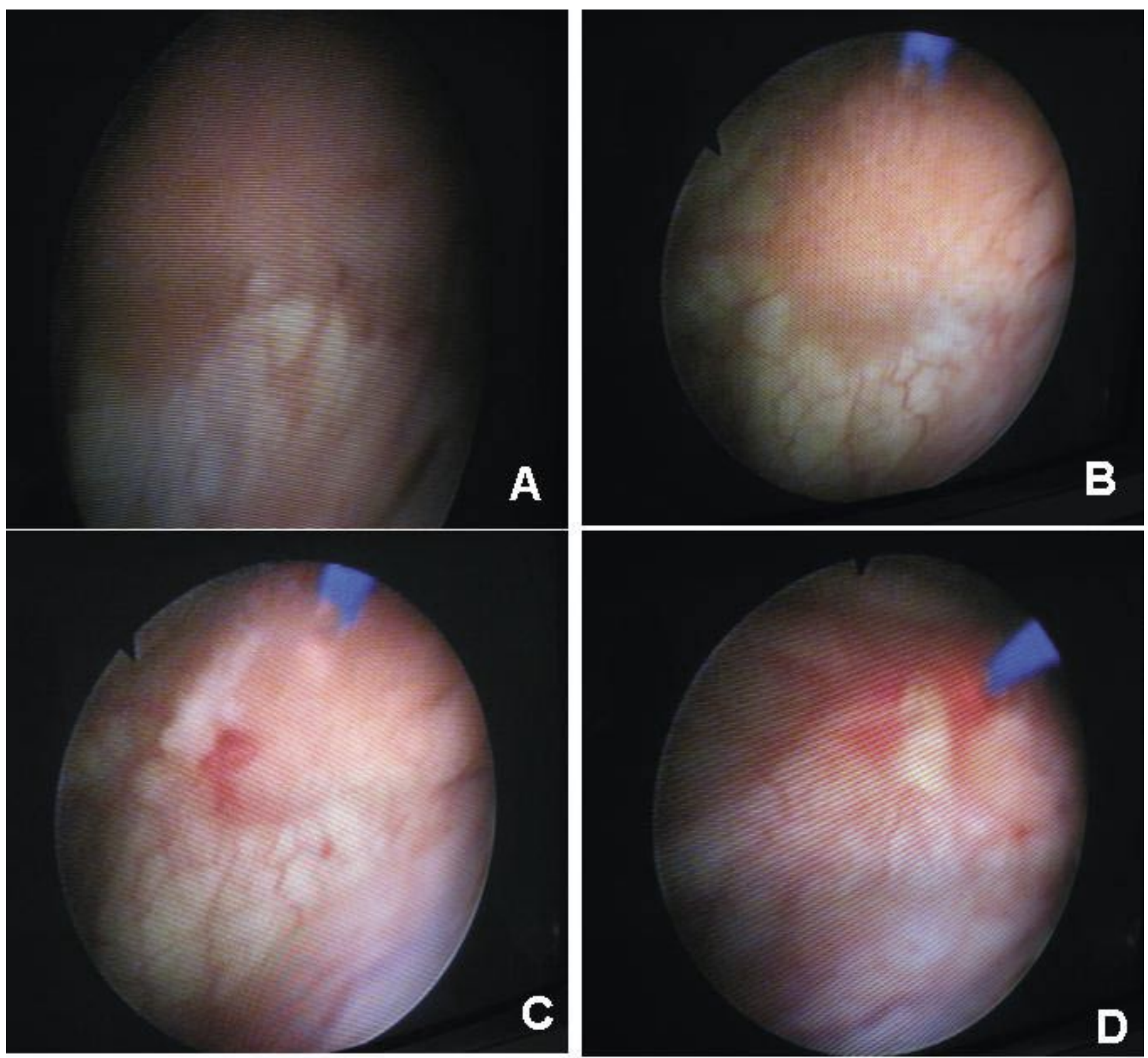

FIGURA 1. Visualización endoscópica de una fotovaporización con láser Holmium de un tumor superficial de vejiga. Al Vista endoscópica de tumor superficial. B) Posicionamiento de fibra láser. C) y D) Fotovaporización del tumor. 
- Cualquier otro tipo de tumor que no sea TCC

- Cualquier estadio tumoral superior o igual a T2

- Lesiones metastásicas

- TCC o CIS en los conductos prostáticos, uréter o uretra

- Porfiria o otra enfermedad hepática grave

- Carotinemia

- Tratamiento con Rifampicina en los últimos 3 meses

- Tratamiento con hidroclorotiazida o productos similares

- Imposibilidad de someterse a anestesia general o raquídea

- Cultivo de orina positivo

- retrasar la cistectomía es la terapia fotodinámica (PDT) (29). El efecto anti-oncogénico de la PDT es secundario a la creación de un gradiente de radicales de oxígeno por activación de un agente foto-sensibilizador en el tejido y de la absorción de determinadas longitudes de onda para un espectro determinado para el agente fotosensibilizador utilizado. La relativa retención selectiva del agente foto-sensibilizador en las células tumorales, resulta en una destrucción más selectiva de las neoplasias, limitando el daño a los tejidos normales adyacentes. Esta cualidad lo convierte en una atractiva opción terapéutica en patologías donde otras técnicas han fracasado (30).

El primer foto-sensibilizador sintético ampliamente utilizado fue un producto derivado de la hemoglobina bo- vina conocido como derivado de la hematoporfirina (HDP), que recientemente se ha refinado en porfirina sódica, un producto resultado de la combinación de esteres y éteres, disponible como Photofrin $®$, el cual ha sido el agente más frecuentemente utilizado en el humano. Más recientemente se ha utilizado el ácido 5-aminolevulínico (5-ALA) tanto de manera oral como tópica como agente sensibilizador (31, 32).

En el campo de la urología, el empleo de la PDT se ha limitado exclusivamente al tratamiento del cáncer superficial de vejiga. La terapia fotodinámica de lesiones papilares presenta resultados diversos, siendo sus resultados más consistentes en el tratamiento del carcinoma in situ (CIS) (33). Uno de los puntos que más atención requiere,
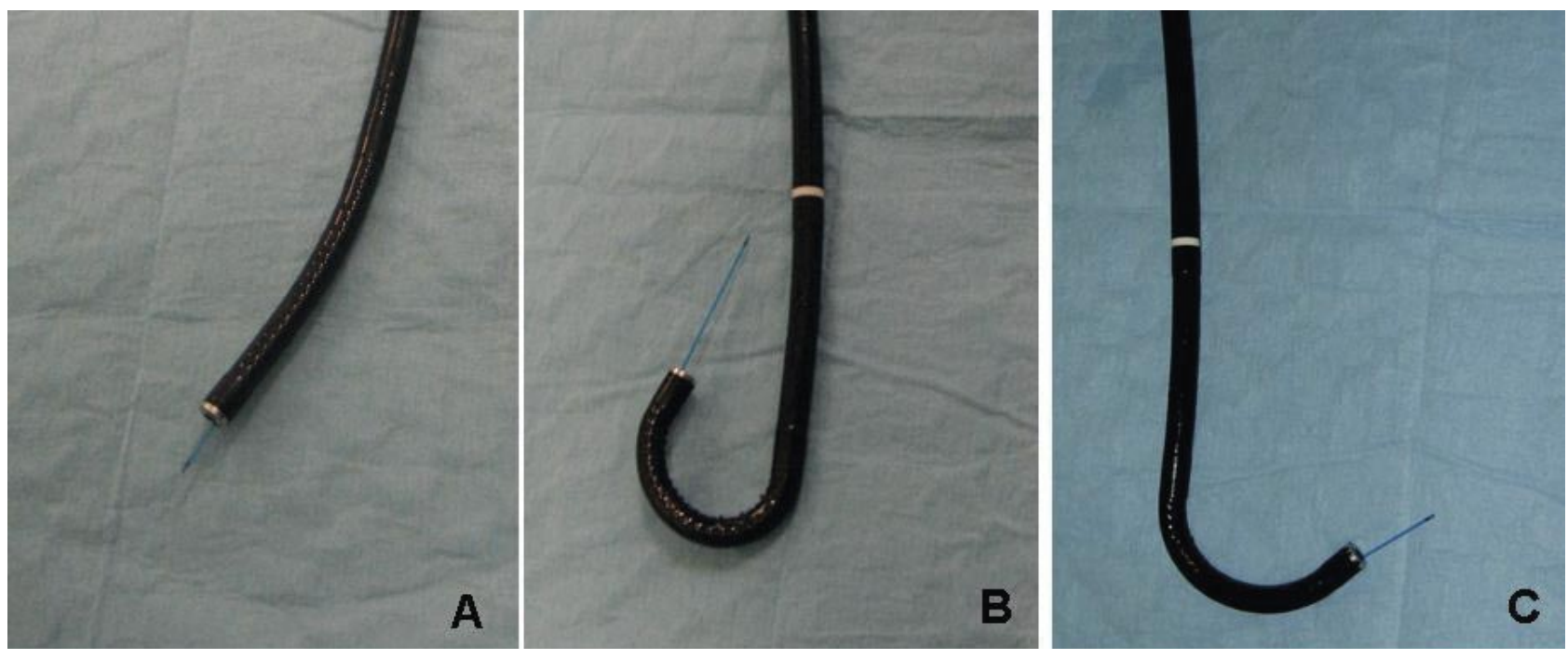

FIGURA 2. Utilización de la fibra láser con endoscopio flexible. Al Posicionamiento de la fibra en el endoscopio con 0 grados de flexión. B) y C) Distintos grados de deflexión del endoscopio con la fibra láser en su interior. 
es el de la dosimetría. Una inadecuada profundidad de penetración de la luz puede conllevar a un fracaso terapéutico en las lesiones papilares. La entrega de energía en órganos huecos es más complicada cuando se intenta tratar áreas más extensas, como en el carcinoma in situ. La punta esférica de la fibra óptica confiere una adecuada distribución de la luz, pero la vejiga no siempre se encuentra simétricamente distendida, lo que conlleva variaciones en la irradiación tisular.

La realización de este tipo de tratamientos es muy compleja y requiere que el paciente cumpla unos determinados requisitos, así como tiene unos criterios de exclusión muy bien definidos (33) (Tabla IV).

Manyak y cols. (33) obtuvieron, empleando porfirina sódica en un medio de difusión intravesical con una fuente de luz de láser KTP:YAG, unos resultado alentadores. Más de la mitad de los pacientes con carcinoma de células transicionales refractario al tratamiento se beneficiaron del tratamiento con PDT. Una revisión reciente de las series publicadas de pacientes tratados con PDT por carcinoma vesical informa de una respuesta completa en el $51 \%$ de los tumores papilares de vejiga y de un $66 \%$ en los casos de $\mathrm{CIS}$ (34).

La mayoría de los pacientes desarrolla algún grado de super-sensibilidad a la luz, por lo que se recomienda evitar la exposición a la luz durante 4 a 6 semanas.

Tan sólo un estudio ha documentado los resultados a largo plazo de un tratamiento único con PDT, evaluando a 15 pacientes a 24 y 54 meses. De estos 9 presentaron una respuesta completa y otros 2 volvieron a serlo después de un retratamiento con PDT (35).

Recientemente se han publicado los resultados en fase I de una terapia combinad, utilizando instilaciones secuenciales de Mitomicina- $C$, con terapia fotodinámica con 5-ALA (36), con el láser KTP:YAG como fuente lumínica. Concluyen que la terapia combinada es segura y bien tolerada, presenta un potencial papel en el control de tumor superficial de difícil manejo, por lo que habrá que esperar los resultados en fase II de este protocolo.

\section{Perspectivas de Futuro}

\section{Espectroscopía RAMAN}

La espectroscopia RAMAN es una técnica óptica que emplea fotones lumínicos inelásticos, dispersos y molecularmente específicos, para interrogar a los tejidos. De manera que cuando un tejido es iluminado con un luz de láser, los fotones interactúan con los puentes moleculares presentes, donando o recibiendo energía del puente, y produciendo un cambio en su estado vibracional. Posteriormente, cuando existe en el tejido, el fotón tiene un nivel de energía alterada, con una longitud de onda distinta de la del láser original.

Este cambio en la energía fotónica se conoce como "Cambio de RAMAN" y se puede medir en número de ondas. Los fotones interactuando con los distintos puentes bioquímico en los tejidos, producen distintos cambios de RAMAN, que juntados constituyen el espectro RAMAN. Este espectro representa una función directa de la composición molecular del tejido y puede por ello, ofrecer una verdadera imagen de la patología.

Múltiples estudios han demostrado cierta variabilidad inter-observador a la hora de graduar y estratificar el TCC (37-39) pero estudios más recientes han demostrado que puede ser utilizada para diferenciar con precisión entre TCC, vejiga normal y cistitis. La técnica puede ser utilizada para categorizar entre bajo y alto grado así como entre no invasivo e invasivo. Estos resultados prometedores alumbran el potencial de la espectroscopia RAMAN y sus múltiples aplicaciones en distintas áreas de la urología. Pudiéndose establecer como una herramienta más de laboratorio, capaz de asistir al patólogo en establecer la patología vesical.

\section{Microdisección láser}

En los últimos 10 años se ha demostrado que un punto clave en el análisis genético de los tumores malignos es la definición histopatológica exacta, así como la separación de las lesiones investigadas. La micro-disección con láser se ha convertido en una herramienta importante, permitiendo el estudio entidades histológicas específicas así como de lesiones preneoplásicas.

La detección fiable de alteraciones tumor-específica, puede estar comprometida por la presencia de lesiones específicas. La combinación de la microdisección y las técnicas de la biología molecular, han sido utilizadas en el estudio de lesiones en el tracto urinario. Permitiendo el aislamiento de pequeñas lesiones pre-neoplásicas conteniendo únicamente varias células, realizando múltiples análisis, que nos han arrojado nuevos conceptos en el proceso cancerígeno del urotelio. La microdisección láser, es una técnica fundamental, que permite establecer la base de la detección de alteraciones moleculares específicas del cáncer de vejiga (40).

\section{Entrega de DNA}

Los tumores de vejiga presentan un acceso transuretral a la vejiga muy sencillo y fácil. La terapia genética ha experimentado muchos avances en los últimos años, estableciendo múltiples métodos para la transferencia de vectores, como el empleo de adenovirus, sin mucho éxito, o la transferencia de plásmidos de DNA. Método que resulta fácil de aplicar pero que presenta unas tasas de transferencia genética bajas. Se han empleado distintas técnicas para aumentar la tasa de trasferencia, como las ondas de choque a través de la cavitación que producen (41).

La implementación de los láseres en los departamentos de urología, ofrece la posibilidad de utilizar su energía como método de transferencia genética. Recientemente, se ha demostrado que utilizando esta energía, la eficacia de transferencia genética era mayor (42, 43). 

raban el $10 \%$

Sin embargo, las tasas de transferencia no supe-

Pero estudios más recientes, en los que se han estudiado distintos tipos de láseres y sus ajustes más adecuado, se ha demostrado una superioridad el Láser Ho:YAG sobre el Nd:YAG. Aumentando la transferencia genética de un $16 \%$ con una frecuencia de láser de $2 \mathrm{~Hz}$. hasta un $40 \%$ con $10 \mathrm{~Hz}$. (44).

\section{CONCLUSIONES}

La terapéutica con láser tiene un papel definido en los tumores de vejiga, para el tratamiento de los tumores superficiales de bajo grado, recurrentes. Disminuyendo el coste de su tratamiento, así como probablemente incrementando su calidad de vida. También existe un papel del láser en el campo de la investigación.

\section{BIBLIOGRAFÍA y LECTURAS RECOMENDADAS ("lectura de interés $y^{* *}$ lectura fundamental)}

1. JEMAL, A.D.; SIEGEL, R.; WARD, E. y cols.: "Cancer Statistics, 2007”. CA Cancer J. Clin., 57: 43, 2007.

2. OOSTERLINCK, W.; VAN DER MEIJDEN, A.; SYLVESTER, R. y cols.: "Guidelines on TaT1 (non-muscle invasive)". EAU Guidelines. Edited by E. A. Urology: European association Urology, 2006.

**3. BRAUSI, M.; COLLETTE, L.; KURTH, K. y cols.: "Variability in the recurrence rate at first follow-up cystoscopy after TUR in stage Ta T1 transitional cell carcinoma of the bladder: A combined analysis of seven EORTC studies". Eur. Urol., 41: 523, 2002.

4. RIOJAZUAZU, J.; ROBLES GARCÍA, J.E.; BERIÁN POLO, J.M.: "Nuevas tecnologías y novedades de futuro en Urología". Libro del Residente de Urología. Edited by J. Castiñeiras. Madrid: GSK, 2007.

5. MILAM, D.F.: "Physical principles of laser energy". Lasers in urology, 3 ed. Edited by J.A. Smith; B.S. Stein; R.C. Benson; St. Louis: Mosby-Year Book, págs. 1-10, 1994.

6. STEIN, B.S.: "Laser-tissue interaction". Lasers in Urologic Surgery, 3 ed. Edited by J.A. Smith; B.S. Stein; R.C. Benson; St. Louis: Mosby-Year Book, págs. 1026, 1994.

*7. PIETROW, P.K.; SMITH, J.A. Jr.: "Laser treatment for invasive and noninvasive carcinoma of the bladder". J. Endourol., 15: 415, 2001.

8. DENSTEDT, J.D.; RAZVI, H.A.; SALES, J.L. y cols.: "Preliminary experience with holmium: YAG laser lithotripsy". J. Endourol., 9: 255, 1995.

9. FRAUNDORFER, M.; CRESSWELL, M.; GILLING, P. y cols.: "Bladder tumor resection with the holmiun laser (abstract 151)". Br. J. Urol., 80: 39, 1997.

10. JOHNSON, D.E.: "Use of the holmium: YAG (Ho: YAG) laser for treatment of superficial bladder carcinoma”. Laser Surg. Med., 14: 213, 1994.
*11. BAGLEY, D.H.: "Ureteroscopic laser treatment of upper urinary tract tumors". J. Clin. Laser Med. Surg., 16: $55,1998$.

*12. BEER, M.; JOCHAM, D.; BEER, A. y cols.: “Adjuvant laser treatment of bladder cancer: 8 years' experience with the Nd: YAG laser 1064nm”. Br. J. Urol., 63: 478, 1989.

13. BEISLAND, H.O.; SELAND, P.: "A prospective randomized study on neodymium-YAG laser irradiation versus TUR in the treatment of urinary bladder cancer". Scand J. Urol. Nephrol., 20: 209, 1986.

14. MALLOY, T.R.; WEIN, A.J.; SHANBERG, A.: "Superficial transtional cell carcinoma of the bladder with neodymiun: YAG laser: A study of the recurrence rate within the first year (Abstract)". J. Urol., 131: 251, 1984.

15. HOLFSTETTER, A.; KRIEGMAIR, M.; BAUMGARTNER, R.: "Evaluation of laser treatment of bladder cancer". Lasers in Urology Surgery. Edited by J.A. Smith; B.S. Stein; R.C. Benson; St. Louis: Mosby-Year Book, págs. 114-125, 1994.

16. DAS, A.; GILLING, P.; FRAUNDORFER, M.: "Holmiun laser resction of bladder tumor (HoLRBT)". Techn. Urol., 4: 12, 1998.

17. SCALETSCKY, R.; MILAN, D.F.; SMITH, J.A.: "Combined laparoscopic and cystoscopic Nd: YAG laser photocoagulation of the porcine bladder wall (abstract)". J. Urol., 149: 411, 1993.

*18. VICENTE, J.; SALVADOR, J.; LAGUNA, P. y cols.: "Laser therapy of superficial tumors of the bladder". Arch. Esp. Urol., 43: 125, 1990.

19. SYED, H.A.; BIYANI, C.S.; BRYAN, N. y cols.: "Holmium: YAG laser treatment of recurrent superficial bladder carcinoma: Initial clinical experience". J. Endourol., 15: 625, 2001.

**20. SOLER-MARTINEZ, J.; VOZMEDIANO-CHICHARRO, R.; MORALES-JIMENEZ, P. y cols.: "Holmium laser treatment for low grade, low stage, noninvasive bladder cancer with local anesthesia and early instillation of mitomycin C'. J. Urol., 178: 2337, 2007.

**21. JONLER, M.; LUND, L.; BISBALLE, S.: "Holmium: YAG laser vaporization of recurrent papillary tumours of the bladder under local anaesthesia”. BJU Int., 94: 322, 2004.

22. CHANG, S.; HALL, M.C.; DALBAGNI, G. y cols. "Treating Non-muscle-invasive Bladder Cancer: The AUA Update". Medscape, 2008.

23. McPHEE, M.S.; ARNFIELD, M.R.; TULIP, J. y cols.: "Neodymium: YAG laser therapy for infiltrating bladder cancer". J. Urol., 140: 44, 1988.

24. SMITH, J.A. Jr.: "Current concepts in laser treatment of bladder cancer". Prog. Clin. Biol. Res., 303: 463 , 1989.

*25. BEISLAND, H.O.; SANDER, S.: "Neodymium-YAG laser irradiation of stage T2 muscle-invasive bladder cancer. Long-term results". Br. J. Urol., 65: 24, 1990.

26. TARANTINO, A.E.; ARETZ, H.T.; LIBERTINO, J.A. y cols.: "Is the neodymium: YAG laser effective therapy for invasive bladder cancer?". Urology, 38: 514, 1991.

27. SANDER, S.; BEISLAND, H.O.: "Superficial bladder cancer". Laser in Urologic Surgery. Edited by J.A. Smith; B.S. Stein; R.C. Benson; St. Louis: Mosby Year Book, págs. 126-134, 1994. 
28. MANYAK, M.J.: "Photodynamic therapy". Laser in Urology Surgery. Edited by J.A. Smith; B.S. Stein; R.C. Benson; St Louis: Mosby Year Book, págs. 230-258, 1994.

29. DOUGHERTY, T.J.: "Photodynamic therapy (PDT) of malignant tumors". Crit. Rev. Oncol. Hematol., 2: 83, 1984.

30. DE REIJKE, T.M.: "Introduction to photodynamic theraphy for superficial bladder carcinoma". The physics of photodynamic theraphy for bladder carcinoma. Edited by H.J. Van Staveren; Amsterdam: UVA, págs. 1-7, 1995.

31. KRIEGMAIR, M.; BAUMGARTNER, R.; LUMPER, W. y cols.: "Early clinical experience with 5-aminolevulinic acid for the photodynamic therapy of superficial bladder cancer". Br. J. Urol., 77: 667, 1996.

*32. WAIDELICH, R.; STEPP, H.; BAUMGARTNER, R. y cols.: "Clinical experience with 5-aminolevulinic acid and photodynamic therapy for refractory superficial bladder cancer". J. Urol., 165: 1904, 2001.

33. MANYAK, M.J.; OGAN, K.: "Photodynamic therapy for refractory superficial bladder cancer: Long-term clinical outcomes of single treatment using intravesical diffusion medium". J. Endourol., 17: 633, 2003.

**34. WALTHER, M.M.: "The role of photodynamic therapy in the treatment of recurrent superficial bladder cancer". Urol. Clin. North Am., 27: 163, 2000.

35. JOCHAM, D.; BEER, M.; BAUMGARTNER, R. y cols.: "Long term experience with integral photodynamyc therapy of Tis bladder carcinoma". Ciba Found Sym., 6: 198, 1989.

36. SKYRME, R.J.; FRENCH, A.J.; DATTA, S.N. y cols.: "A phase-1 study of sequential mitomycin $\mathrm{C}$ and 5aminolaevulinic acid-mediated photodynamic therapy in recurrent superficial bladder carcinoma". BJU Int., 95: 1206, 2005.

37. OOMS, E.C.; ANDERSON, W.A.; ALONS, C.L. y cols.: "Analysis of the performance of pathologists in the grading of bladder tumors". Hum. Pathol., 14: 140, 1983.

38. BOL, M.G.; BAAK, J.P.; BUHR-WILDHAGEN, S. y cols.: "Reproducibility and prognostic variability of grade and lamina propria invasion in stages Ta, T1 urothelial carcinoma of the bladder". J. Urol., 169: 1291, 2003.

39. TOSONI, I.; WAGNER, U.; SAUTER, G. y cols.: "Clinical significance of interobserver differences in the staging and grading of superficial bladder cancer". BJU Int., 85: 48, 2000.

40. STOEHR, R.; WILD, P.; HARTMANN, A.: "Lasermicrodissection: An Important Prerequisite for the Molecular-genetic analysis of bladder cancer". Pathol. Res. Pract., 199: 355, 2003.

41. MICHEL, M.S.; SCHAAF, A.; KÖHRMANN, K.U. y cols.: "Transfection of transitional cell carincoma by extracorporeal acoustical energy: A new method for gene therapy of localised bladder tumors?". 167: 159, 2002.

*42. TIRLAPUR, U.K.; KONIG, K.: "Targeted transfection by femtosecond laser". Nature, 418: 290, 2002.

43. SHIRAHATA, Y.; OHKOHCHI, N.; ITAGAK, H. y cols.: "New technique for gene transfection using laser irradiation". J. Investig. Med., 49: 184, 2001.

44. KNOLL, T.; TROJAN, L.; LANGBEIN, S. y cols.: "Impact of holmium: YAG and neodymium: YAG lasers on the efficacy of DNA delivery in transitional cell carcinoma". Laser in Medical Science, 19: 33, 2004. 